\title{
The impact of an unpredictable context and intolerance of uncertainty on the electrocortical response to monetary gains and losses
}

\author{
Brady D. Nelson ${ }^{1} \cdot$ Ellen M. Kessel $^{1} \cdot$ Felicia Jackson $^{1} \cdot$ Greg Hajcak $^{1}$
}

Published online: 5 October 2015

(C) Psychonomic Society, Inc. 2015

\begin{abstract}
There is growing evidence that unpredictability and uncertainty can alter reward system functioning. The present study examined the impact of (1) a task-irrelevant unpredictable relative to predictable context and (2) individual differences in intolerance of uncertainty (IU) on the reward-related positivity (RewP), an event-related potential (ERP) response to monetary gains relative to losses. Specifically, 64 participants listened to predictable and unpredictable tone sequences while electroencephalography was recorded during a monetary gambling task. Participants also completed the Intolerance of Uncertainty Scale, which measures both cognitive distress (prospective IU) and behavioral inhibition (inhibitory IU) elicited by uncertainty, in addition to the Depression Anxiety Stress Scale-21 and Penn State Worry Questionnaire. Results indicated that the RewP was reduced during the unpredictable relative to the predictable context. Greater selfreported anxiety elicited by the unpredictable context was associated with a decreased RewP, and a decreased RewP was associated with poorer lose-shift behavioral adjustment. Furthermore, the RewP mediated the relationship between selfreported anxiety elicited by the unpredictable context and lose-shift behavioral adjustment. The IU subscales demonstrated the opposite relationship with the RewP across both contexts - inhibitory IU was associated with an attenuated RewP and prospective IU was associated with an enhanced RewP. In contrast, anxiety, depression, stress, and worry symptomatology were not associated with the RewP. This is the first study to demonstrate that an unpredictable context
\end{abstract}

Brady D. Nelson

brady.nelson@stonybrook.edu

1 Department of Psychology, Stony Brook University, Stony Brook, NY, USA and individual differences in the degree to which people cannot tolerate uncertainty impact an ERP measure of reward system functioning.

Keywords Event-related potentials · Intolerance of uncertainty $\cdot$ Reward $\cdot$ Unpredictability

The ability to predict salient information in the environment is critical for survival. For example, people prefer predictable over unpredictable threat (Grillon, Baas, Cornwell, \& Johnson, 2006; Lejuez, Eifert, Zvolensky, \& Richards, 2000), as predictability allows for better preparation and action (Grupe $\&$ Nitschke, 2013). Furthermore, an emerging literature suggests that an unpredictable context itself may contribute to heightened sensitivity to threat (Herry et al., 2007; Jackson, Nelson, \& Proudfit, 2015a).

The preference for predictability has implications beyond threat detection. Individuals tend to seek out a predictable environment (Heine, Proulx, \& Vohs, 2006) because an unpredictable environment makes the world appear less ordered and organized. Theoretical models propose that the degree to which an environment is perceived as predictable and ordered also influences the facilitation of approach behavior (HarmonJones \& Harmon-Jones, 2002; Lerner, 1980; McGregor, Prentice, \& Nash, 2009). These theories suggest that unpredictability within one's milieu is stressful because it is more challenging to learn to anticipate pleasant events due to actions and outcomes being less tightly linked.

There is growing evidence that the anxiety and stress elicited by unpredictability disrupts reward system functioning. For example, animal studies have indicated that rats exposed to chronic unpredictable mild stress demonstrate decreased sensitivity to reward (Papp, Willner, \& Muscat, 1991; Willner, Towell, Sampson, Sophokleous, \& Muscat, 1987). In humans, individuals with a history of childhood adversity, an environmental context characterized by both extreme levels of 
harshness and unpredictability, demonstrate reduced activation in the left basal ganglia - a brain region implicated in reward learning and motivation - compared to controls (Dillon et al., 2009). Taken together, these studies suggest that an unpredictable stressful environment can lead to long-lasting deficits in reward processing. However, these studies confound the influence of a harsh environment and unpredictability per se, and it is unclear whether an unpredictable context in and of itself impacts reward system functioning.

Recent work has manipulated contextual predictability in the laboratory by exposing individuals to repetitive auditory tone sequences that occur at either predictable or unpredictable temporal intervals. Herry et al. (2007) found that a task-irrelevant unpredictable tone sequence potentiated amygdala activation and increased attentional bias to threatening stimuli. Moreover, Jackson et al. (2015a) found that the unpredictable compared to predictable tone sequence enhanced the neural response to errors (i.e., the error-related negativity, or ERN). Together, these findings suggest that a task-irrelevant unpredictable context can impact neural and behavioral measures of threat processing. The present study tested whether the same contextual manipulation influenced reward system functioning.

The event-related potential (ERP) has been used extensively to study neural activity in response to monetary gains and losses. Approximately $300 \mathrm{~ms}$ after feedback, the ERP at frontocentral recording sites is characterized by a relative negativity following losses (Hajcak, Moser, Holroyd, \& Simons, 2006; Holroyd, Hajcak, \& Larsen, 2006). The ERP difference between gains and losses was originally conceptualized in terms of a negative deflection in the ERP following unfavorable feedback (i.e., the feedback negativity, or FN; Hajcak, Moser, Holroyd, \& Simons, 2007; Yeung, Holroyd, \& Cohen, 2005). However, recent evidence suggests that the relative difference between gains and losses could reflect two distinct components with comparable temporal properties: a negativity in response to losses and a positivity in response to rewards that is reduced or absent for losses (Holroyd, Pakzad-Vaezi, \& Krigolson, 2008; Proudfit, 2015). The ERP response to losses has been linked to activation in the anterior cingulate cortex (Dehaene, Posner, \& Tucker, 1994; Holroyd, Dien, \& Coles, 1998; Miltner, Braun, \& Coles, 1997). In contrast, the ERP response to gains has been associated with behavioral measures of reward sensitivity (Bress \& Hajcak, 2013) and activation in key reward-related regions, including the ventral striatum and medial prefrontal cortex (mPFC; Carlson, Foti, Mujica-Parodi, Harmon-Jones, \& Hajcak, 2011; Foti, Carlson, Sauder, \& Proudfit, 2014). These data have led several researchers to refer to the difference between gains and losses as the reward-related positivity (RewP; Holroyd et al., 2008; Proudfit, 2015).

The present study examined the impact of a task-irrelevant unpredictable context on the RewP. Specifically, 64 participants listened to predictable and unpredictable tone sequences while electroencephalography (EEG) was recorded during a monetary gambling task, and the RewP was measured in response to equiprobable gains and losses. Win-stay and loseshift behavioral adjustments were also calculated after completion of the gambling task. The experimental design allowed us to examine the impact of a task-irrelevant unpredictable vs. predictable context (i.e., tone sequences) on behavioral and neural measures of task-relevant reinforcement learning during a reward context (i.e., gambling task). Given that previous research has shown that an unpredictable harsh environment diminishes reward system functioning (Dillon et al., 2009; Pryce, Rüedi-Bettschen, \& Dettling, 2005), we hypothesized that the unpredictable, relative to predictable, context would decrease the RewP and both win-stay and lose-shift behavioral adjustments.

It is also possible that individuals who are more sensitive to uncertainty would be more susceptible to the effect of an unpredictable context on reward system functioning. For example, one study found that, in individuals with commensurate levels of stress exposure, only those who appraised their environment as being uncertain and unpredictable exhibited decreased reward responsiveness (Pizzagalli, Bogdan, Ratner, \& Jahn, 2007). Furthermore, a separate investigation found that participants reporting more unpredictable and uncontrollable stressors displayed blunted neural responses in medial PFC (Treadway, Buckholtz, \& Zald, 2013).

Intolerance of uncertainty (IU) is the tendency to perceive, interpret, and respond to ambiguous or uncertain situations as aversive and stressful (Dugas, Buhr, \& Ladouceur, 2004). IU has been associated with several anxiety disorders (e.g., generalized anxiety disorder [GAD], obsessive-compulsive disorder [OCD]; Dugas, Gagnon, Ladouceur, \& Freeston, 1998; Tolin, Abramowitz, Brigidi, \& Foa, 2003) and depression (Carleton et al., 2012), and treatments targeting IU reduce symptoms of anxiety and depression (Robichaud, 2013; van der Heiden, Muris, \& van der Molen, 2012). These findings have led several researchers to suggest that IU constitutes a transdiagnostic mechanism of emotional disorders (Boswell, Thompson-Hollands, Farchione, \& Barlow, 2013; Mahoney $\&$ McEvoy, 2012). The present study also examined whether individual differences in IU were associated with the RewP and behavioral adjustment during the unpredictable and predictable contexts. Since individuals with high IU find unpredictably to be aversive and stressful, we hypothesized that greater IU would be associated with a decreased RewP and poorer behavioral adjustment during the unpredictable (but not predictable) context.

Finally, it is important to highlight that IU is characterized by two related, but distinct, factors: inhibitory IU and prospective IU (Birrell, Meares, Wilkinson, \& Freeston, 2011). Inhibitory IU represents behavioral inhibition and paralysis in the face of uncertainty, whereas prospective IU characterizes cognitive concerns about uncertain future events (Carleton, Norton, \& Asmundson, 2007). A number of studies have identified 
distinct relationships between inhibitory and prospective IU and psychobiological responding to uncertainty. For example, inhibitory IU has been associated with a decreased startle reflex while anticipating uncertain threat (Nelson \& Shankman, 2011) and an attenuated ERN (Jackson, Nelson, \& Hajcak, 2015b), whereas prospective IU has been associated with an increased startle reflex while anticipating uncertain threat (Nelson, Liu, Sarapas, \& Shankman, 2015) and an enhanced ERN (Jackson et al., 2015b). Therefore, as an exploratory analysis, we examined whether there were specific relationships between the inhibitory and prospective IU subscales and the RewP. Furthermore, we also assessed self-reported anxiety, depression, stress, and worry to determine whether current internalizing symptoms, which are highly correlated with IU, demonstrated a similar relationship with reward system functioning across the predictable and unpredictable contexts.

\section{Method}

\section{Participants}

Sixty-four undergraduates from Stony Brook University participated for course credit. The sample was predominately female $(85.7 \%)$, college-aged $(M=20.43$ years, $S D=3.03)$, and ethnically diverse, including $30.2 \%$ Caucasian, $41.3 \%$ Asian, 7.9\% Black, 17.5\% Latino, and 3.2\% "Other." No participants were currently taking psychiatric medication. Informed consent was obtained prior to participation and the research protocol was approved by the Institutional Review Board at Stony Brook University.

\section{Measures}

Intolerance of Uncertainty Scale (IUS) The IUS (Freeston, Rhéaume, Letarte, Dugas, \& Ladouceur, 1994) is a 27-item self-report measure that assesses the degree to which individuals find uncertainty to be distressing, frustrating, and undesirable. Items are rated on a 5-point Likert scale ranging from 1 (not at all characteristic of me) to 5 (entirely characteristic of $m e$ ), with higher scores indicating a greater IU. The present study utilized the more parsimonious 12-item version of the IUS (Carleton et al., 2007), which excludes items specific to GAD and demonstrates improved psychometric properties. The 12 -item IUS $(M=27.60, S D=8.46)$ produces two factor-analytically derived subscales: a 5-item inhibitory IU subscale $(M=9.93, S D=3.48)$ measuring the propensity for uncertainty to inhibit one's actions or experiences, and a 7-item prospective IU subscale $(M=17.67, S D=5.49)$ measuring fear and anxiety in response to future events. As expected, the IU subscales were moderately correlated, $r(55)=.77, p<.001$.
Depression Anxiety Stress Scales-21 (DASS-21) The DASS-21 is a measure of psychological distress over the last week (Lovibond \& Lovibond, 1995). Items are rated on a 4point Likert scale ranging from 0 (did not apply to me at all) to 4 (applied to me very much, or most of the time), with higher scores indicating greater symptom severity. The DASS-21 was designed in accordance with the tripartite model of depression and anxiety (Clark \& Watson, 1991), and contains three 7-item subscales measuring low positive affect (DASSDepression; $M=3.93, S D=4.12$ ), physiological hyperarousal (DASS-Anxiety; $M=3.16, S D=3.12$ ), and nonspecific negative affect (DASS-Stress; $M=5.11, S D=4.09$ ).

Penn State Worry Questionnaire (PSWQ) The PSWQ (Meyer, Miller, Metzger, \& Borkovec, 1990) is a 16-item self-report measure that assesses the trait-like tendency to engage in excessive, uncontrollable, and generalized worry. Items are rated on a 5-point Likert scale ranging from 1 (not at all typical) to 5 (very typical), with higher scores indicate a greater tendency to worry $(M=41.56, S D=9.27)$.

\section{Stimuli}

Predictable and unpredictable tones were identical to those used in Herry et al. (2007) and Jackson et al. (2015a). The carrier frequency was $1 \mathrm{kHz}$ with pulse duration of $40 \mathrm{~ms}$ and mean pulse spacing of $200 \mathrm{~ms}$ ( $5 \mathrm{~Hz}$ pulse repetition rate). The unpredictable tone sequence was generated from the predictable sequence by randomly varying the intertone interval. The randomly selected temporal shift was confined to an interval $\sigma$ with uniform probability within $\sigma$. In the unpredictable sequence $\sigma$ was set to $120 \mathrm{~ms}$, and in the predictable sequence $\sigma$ was set to $0 \mathrm{~ms}$. Thus, the predictable and unpredictable sequences contained the same number of tones with equivalent mean interstimulus interval. The tones were not timelocked to any event because each trial of the monetary gambling task (i.e., the doors task, see below) did not proceed until the participant chose a door. This elicited a variable intertrial interval and prevented the tone sequences from being synched with any particular aspect of the task. The sequences were played at $85 \mathrm{~dB}$ through external computer speakers positioned approximately $50 \mathrm{~cm}$ in front of the participant.

\section{Procedure}

After written informed consent was obtained, EEG was recorded, and participants completed the doors task while listening to the unpredictable or predictable tone sequences in an alternating order (counterbalanced). After completing the reward task, the EEG electrodes were removed and participants completed the self-report questionnaires. 
Doors task The doors task was administered using Presentation software (Neurobehavioral Systems, Inc., Albany, CA, USA) and was similar to the version used in previous studies (Proudfit, 2015). The task consisted of 80 trials presented over four blocks of 20 trials. During each block, either the predictable (P) or unpredictable $(\mathrm{U})$ tones were played through computer speakers. Tone block order (UPUP or PUPU) was counterbalanced.

Each trial began with the presentation of two identical doors. Participants were instructed to select the left or right door by clicking the left or right mouse button, respectively. Participants were told that they could either win $\$ 0.50$ or lose $\$ 0.25$ on each trial. These values were chosen in order to equalize the subjective value of the gains and losses (Tversky \& Kahneman, 1981; Tversky \& Kahneman, 1992). The goal of the task was to guess which door hid the reward while attempting to earn as much money as possible. The image of the doors was presented until the participant made a selection. After stimulus offset, a fixation cross $(+)$ was presented for 1 , $000 \mathrm{~ms}$, and feedback was then presented on the screen for 2 , $000 \mathrm{~ms}$. A gain was indicated by a green arrow pointing upward $(\uparrow)$, and a loss was indicated by a red arrow pointing downward $(\downarrow)$. The feedback stimulus was followed by a fixation cross presented for 1,500 ms, immediately followed by the message "Click for next round." This prompt remained on the screen until the participant responded with a button press to initiate the next trial. There were an equal number of gain and loss trials (40 each), such that participants had an equal likelihood of receiving gain and loss feedback throughout the task, and the frequency of gain and loss trials were balanced across predictable and unpredictable tone conditions.

At the end of the doors task, participants rated their anxiety during the predictable and unpredictable blocks on a 7-point Likert scale ranging from 1 (not anxious) to 7 (extremely anxious). Participants were also asked to indicate which tone sequence they disliked the most (predictable or unpredictable). The tones were presented again at the end of the task to confirm participants correctly labeled them during the subjective ratings.

The doors task also provided the opportunity to examine behavioral adjustment following gain and loss trials. Specifically, we examined the percentage of trials where participants chose to win-stay (i.e., choosing the same door following a gain) and lose-shift (i.e., choosing the opposite door following a loss). Separate win-stay and lose-shift percentages were calculated for trials that occurred during the predictable and unpredictable contexts.

EEG recording and processing Continuous EEG was recorded using an elastic cap with 34 electrode sites placed according to the $10 / 20$ system. Electrooculogram (EOG) was recorded using four additional facial electrodes: two placed approximately $1 \mathrm{~cm}$ outside of the right and left eyes and two placed approximately $1 \mathrm{~cm}$ above and below the right eye. All electrodes were sintered $\mathrm{Ag} / \mathrm{AgCl}$ electrodes. Data were recorded using the Active Two BioSemi system (BioSemi, Amsterdam, Netherlands). The EEG was digitized with a sampling rate of $1024 \mathrm{~Hz}$ using a low-pass fifth order sinc filter with a half-power cutoff of $204.8 \mathrm{~Hz}$. A common mode sense active electrode producing a monopolar (nondifferential) channel was used as recording reference.

EEG data were analyzed using Brain Vision Analyzer (Brain Products, Gilching, Germany). Data were referenced offline to the average of left and right mastoids, band-pass filtered $(0.1$ to $30 \mathrm{~Hz})$, and corrected for eye movement artifacts (Gratton, Coles, \& Donchin, 1983). Feedback-locked epochs were extracted with a duration of 1,000 ms, including a $200 \mathrm{~ms}$ prestimulus and $800 \mathrm{~ms}$ poststimulus interval. The $200 \mathrm{~ms}$ prestimulus interval was used as the baseline. Epochs containing a voltage greater than $50 \mu \mathrm{V}$ between sample points, a voltage difference of $300 \mu \mathrm{V}$ within a segment, or a maximum voltage difference of less than $0.50 \mu \mathrm{V}$ within $100 \mathrm{~ms}$ intervals were automatically rejected. Additional artifacts were identified and removed based on visual inspection.

Feedback-locked ERPs were averaged separately for gains and losses. The ERP response to gains and losses were separately scored as the mean amplitude from 250 to $350 \mathrm{~ms}$ following feedback at $\mathrm{FCz}$, where the difference between gains and losses was maximal. The RewP was then quantified as the difference between gain and loss trials (i.e., gain-loss).

\section{Data analysis}

Nine participants were excluded from analyses due to equipment failure $(n=2)$ or excessive EEG artifacts that resulted in less than $50 \%$ usable trials $(n=7)$, leaving a final sample of 55 participants. A repeated measures analysis of variance (ANOVA) with condition (predictable vs. unpredictable) as a within-subjects factor was conducted to examine the impact of the predictable and unpredictable tones on the RewP. Pearson's correlations were conducted to examine the association between self-reported anxiety, the RewP, and behavioral adjustments. Separate correlations were conducted for the measures collected during the predictable and unpredictable context. A mixed-measures analysis of covariance (ANCOVA) with condition as a within-subjects factor and IU as a mean-centered covariate was conducted to examine the impact of IU on the RewP. Identical analyses were conducted for the DASS-21 subscales and the PSWQ. All analyses were conducted in IBM SPSS Statistics, Version 22.0 (Armonk, NY, USA).

\section{Results}

\section{Self-report ratings}

Participants reported greater anxiety during the unpredictable $(M=3.55, S D=1.61)$ relative to the predictable context $(M=$ 
2.87, $S D=1.47), t(54)=3.46, p<.001$. Furthermore, more participants reported disliking the unpredictable $(63.6 \%)$ relative to predictable context $(36.4 \%), t(54)=9.72, p<.001$.

\section{ERPs}

As expected, across both the predictable and unpredictable contexts the ERP response to monetary gains $(M=17.78$, $S D=7.36$ ) was greater (i.e., more positive) than the response to losses $(M=12.53, S D=12.53), F(1,54)=67.83, p<.001$, $\eta_{\mathrm{p}}{ }^{2}=.56$. As shown in Fig. 1, the RewP (i.e., ERP response to gains-losses) was decreased (i.e., less positive) during the unpredictable relative to the predictable context, $F(1,54)=$ $4.07, p<.05, \eta_{\mathrm{p}}^{2}=.07$. We also examined whether there were condition differences between the ERP response to gains and losses alone. Results indicated the ERP response to gains, $F(1$, $54)=1.07, n s$, and losses, $F(1,54)=0.88, n s$, alone did not differ between conditions. Thus, the unpredictable tones appeared to reduce the relative difference between the ERP response to gains and losses (i.e., the RewP), and did not specifically impact either component alone. ${ }^{1}$

Next, we examined the association between self-reported anxiety during the different contexts and the RewP. As shown in Fig. 2 (top), greater self-reported anxiety during the unpredictable context was associated with a decreased RewP during the unpredictable context, $r(55)=-.28, p<.05$; however, there was no relationship between these same measures during the predictable context, $r(55)=.12, n s$. Furthermore, a Fisher $r$-to- $z$ transformation indicated these correlations significantly differed, $z=-2.08, p<.05$, two-tailed. Together, these results suggest that a task-irrelevant unpredictable context decreased the RewP, and this decrement was greatest in individuals who reported increased anxiety during the unpredictable context.

For the IU analyses, we first examined the association between total IU and the RewP. Results indicated no main effect or interaction involving total IU ( $p$ s > .94). Next, we conducted identical analyses, but instead included inhibitory IU and

\footnotetext{
${ }^{1}$ We also tested the impact of a task-irrelevant unpredictable context and IU on neural activity in anticipation of monetary gains and losses. To this end, we examined the stimulus-preceding negativity (SPN), a slow wave cortical potential that is enhanced in anticipation of pleasant or unpleasant relative to neutral stimuli (Poli, Sarlo, Bortoletto, Buodo, \& Palomba, 2007), during the fixation cross that followed the door selection and preceded the response feedback. Stimulus-locked epochs were extracted with a duration of $1,000 \mathrm{~ms}$ and a $0-200 \mathrm{~ms}$ interval baseline. The SPN was scored as the mean amplitude from $800-1,000 \mathrm{~ms}$ following stimulus onset at $\mathrm{FCz}$

A repeated-measures ANOVA indicated the SPN did not differ between the predictable and unpredictable context, $F(1,54)=0.41, n s$. Furthermore, total IU, prospective IU (controlling for inhibitory IU), and inhibitory IU (controlling for prospective IU) were not associated with the SPN during either the predictable or unpredictable context ( $p$ s $>.36$ ). Together, these results suggest that the task-irrelevant unpredictable context and individual differences in IU were not associated with the SPN.
}

prospective IU as simultaneous covariates. Results indicated main effects of inhibitory IU, $F(1,52)=5.43, p<.05, \eta_{\mathrm{p}}{ }^{2}=$ .10 , and prospective IU, $F(1,52)=4.63, p<.05, \eta_{\mathrm{p}}{ }^{2}=.08$; however, there were no interactions between condition and inhibitory IU, $F(1,52)=0.75$, $n s$, or prospective IU, $F(1,52)$ $=0.73, n s$, suggesting that the association between the IU subscales and the RewP did not differ between the predictable or unpredictable contexts. ${ }^{2}$ The IU main effects were follow-up by conducting partial correlations between the IU subscale, controlling for the other subscale, and the RewP averaged across both predictable and unpredictable contexts. As shown in Fig. 3, results indicated the IU subscales demonstrated the opposite relationship with the RewP, such that inhibitory IU was associated with an attenuated RewP, $p r(52)=.31, p<$ .05 , while prospective IU was associated with an enhanced RewP, $p r(52)=-.29, p<.05$. Identical analyses for the DASS subscales and PSWQ yielded no main effects or interactions involving these measures in relation to the $\operatorname{RewP}(p \mathrm{~s}>.18)$.

\section{Behavior}

A one-sample $t$ test was conducted for the win-stay ( $M=$ $56.80 \%, S D=20.15)$ and lose-shift $(M=58.24 \%, S D=$ 18.33 ) percentages averaged across the predictable and unpredictable contexts to determine whether they differed from random chance responding (i.e., 50\%). Results indicated that both win-stay, $t(54)=2.50, p<.05$, and lose-shift, $t(54)=$ $3.34, p<.01$, percentages differed from $50 \%$, suggesting participants attempted to adjust their behavior based on task performance. However, win-stay and lose-shift percentages were not correlated, $r(55)=.11, n s$.

Next, we examined the association between the RewP and behavioral adjustment. As shown in Fig. 2 (bottom), the RewP was positively correlated with lose-shift behavioral adjustment during the unpredictable context, $r(55)=.30, p<.05$. To determine whether this association was primarily due to the electrocortical response to monetary gains or losses, we conducted additional correlations between the ERP response to gains and losses alone and lose-shift behavioral adjustment. Results indicated that the ERP response to gains was positively associated with lose-shift behavioral adjustment at a trend level, $r(55)=.24, p<.08$, but there was no association between the ERP response to losses and lose-shift behavioral adjustment, $r(55)=.06, n s$. Thus, the association between the RewP and lose-shift behavioral adjustment was primary driven by the electrocortical response to monetary gains. There was no association between the RewP and win-stay percentage during the unpredictable context or the RewP and either behavioral adjustment during the predictable context $(p s>$

\footnotetext{
${ }^{2}$ When separate Condition $\times$ IU analyses were conducted for prospective and inhibitory IU, there were no main effects or interactions for either IU subscale $(p s>.31)$.
} 


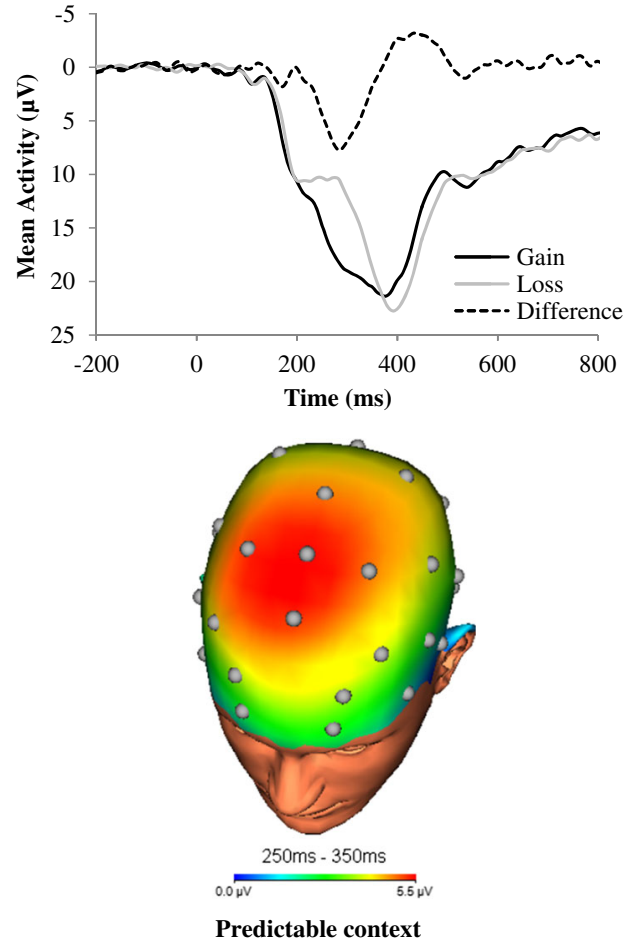

Fig. 1 Waveforms and 3-D rendered scalp topographies of the ERP response to monetary gains, losses, and their difference (i.e., RewP) during the predicable (left) and unpredictable (right) contexts. ERP =

.10). These result suggest that a reduced RewP was associated with decreased lose-shift behavioral adjustment during the unpredictable context.

There was no bivariate association between self-report anxiety and lose-shift behavioral adjustment during the unpredictable context, $r(55)=.17, n s$. However, we tested for the presence of an indirect effect of anxiety, mediated though the RewP, on lose-shift behavioral adjustment. To this end, the present study used a nonparametric bootstrapping method (MacKinnon, Lockwood, \& Williams, 2004), which has been shown to be statistically more powerful than other tests of mediation (MacKinnon, Lockwood, Hoffman, West, \& Sheets, 2002). Specifically, we used the SPSS macro PROCESS provided by Preacher and Hayes (Hayes, 2013; Preacher \& Hayes, 2004), which provides a bootstrap estimate of the indirect effect between the independent variable and dependent variable, an estimated standard error, and 95\% confidence intervals (CI) for the population value of the indirect effect. CIs for the indirect effect that do not include zero indicate a significant indirect effect at the $p<.05$ significance level. Analyses were conducted using 5,000 bootstrap samples. Prior to conducting mediational analyses, all variables were $z$-scored to produce standardized $\beta$ weights.

As shown in Fig. 4, during the unpredictable context greater self-reported anxiety was associated with a decreased RewP, and, in turn, a decreased RewP was associated with

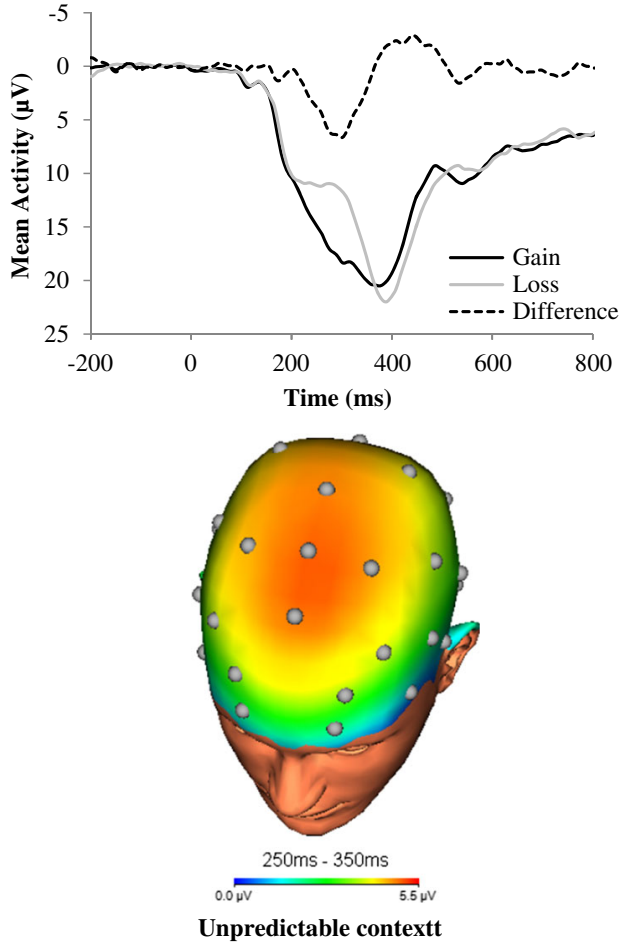

event-related potential; $\mathrm{ms}=$ milliseconds; RewP $=$ reward-related positivity

decreased lose-shift behavioral adjustment. Greater selfreported anxiety was directly associated with increased loseshift behavioral adjustment; however, there was an indirect effect of self-reported anxiety, mediated through the RewP, on behavioral adjustment. Together, these results suggest that, when controlling for the RewP, greater anxiety was associated with increased lose-shift behavioral adjustment, but greater anxiety was also indirectly associated with decreased behavioral adjustment when mediated by a reduced RewP. Finally, given the cross-sectional nature of the mediation model, we also ran a model in which the mediator and the dependent variable were switched. Unlike the previous model, there was no indirect effect of self-reported anxiety on the RewP mediated through lose-shift behavioral adjustment, 95\% CI $[-.02, .20]$.

\section{Discussion}

The present study examined the impact of a task-irrelevant unpredictable context and individual differences in IU on the ERP response to monetary gains relative to losses (i.e., the RewP). Results indicated that the RewP was decreased during the unpredictable relative to the predictable context. In addition, greater self-reported anxiety elicited by the unpredictable context was associated with a decreased RewP, and the 

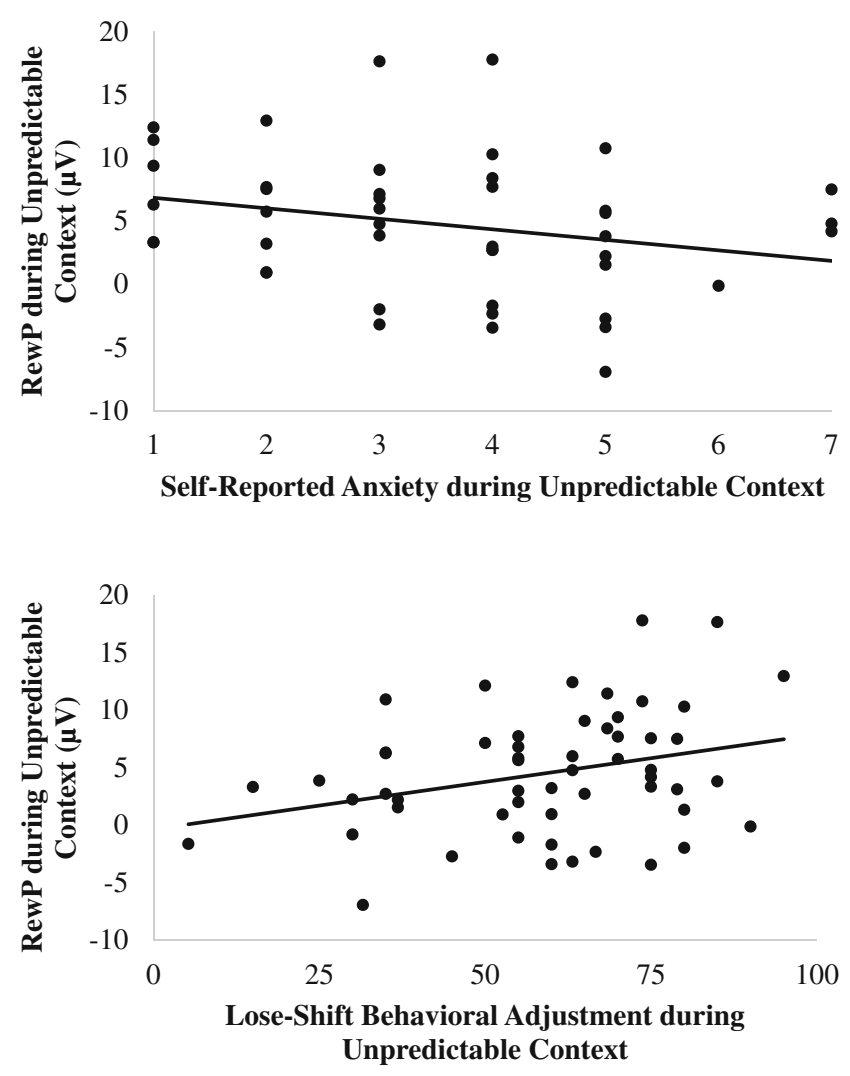

Fig. 2 Scatterplots depicting the association between the RewP (i.e., gain-loss) and self-reported anxiety (top) and lose-shift behavioral adjustment (bottom) during the unpredictable context. IU = intolerance of uncertainty; RewP = reward-related positivity

decreased RewP mediated the relationship between selfreported anxiety and lose-shift behavioral adjustment. These results are consistent with previous investigations showing that a harsh environment decreases reward system functioning (Dillon et al., 2009; Papp et al., 1991; Willner et al., 1987). In contrast to our hypothesis, greater IU was not associated with increased susceptibility to the effect of a task-irrelevant unpredictable context on reward system functioning. However, the
IUS subscales demonstrated the opposite relationship with the RewP across both predictable and unpredictable contexts. Specifically, inhibitory IU was associated with an attenuated RewP and prospective IU was associated with an enhanced RewP. Finally, there were no associations between internalizing symptoms (anxiety, depression, stress, and worry) and the RewP across either the predictable or unpredictable context.

The task-irrelevant unpredictable, relative to predictable, context was rated as more anxiety-provoking and decreased the RewP. Furthermore, greater self-reported anxiety elicited by the unpredictable context was associated with a reduced RewP, while this relationship was not present for the predictable context. Previous investigations have found that the exact same unpredictable tone sequence enhances amygdala activation and attentional bias to threat (Herry et al., 2007) and errorrelated brain activity (Jackson et al., 2015a). Therefore, in the present study it is possible that the unpredictable context primed defense system activation, which in turn interfered with reward processing. This is consistent with previous research demonstrating that threat processing trumps reward processing (Frewen, Dozois, Joanisse, \& Neufeld, 2008), and research indicating that stress can alter dopaminergic functioning (Lemos et al., 2012) and diminish both neural sensitivity to reward (Porcelli, Lewis, \& Delgado, 2012) and reward learning (Bogdan \& Pizzagalli, 2006). Overall, the present study suggests that a task-irrelevant unpredictable context has the capacity to alter a neural measure of reward system functioning.

Contrary to our prediction, high IU individuals were not more susceptible to the effects of a task-irrelevant unpredictable context on reward system functioning. However, the inhibitory and prospective IU dimensions were associated with an attenuated and enhanced RewP, respectively, across both the predictable and unpredictable contexts. Inhibitory IU has been associated with a decreased startle reflex in anticipation of unpredictable electric shocks (Nelson \& Shankman, 2011) and neural response to errors (i.e., the ERN; Jackson et al.,

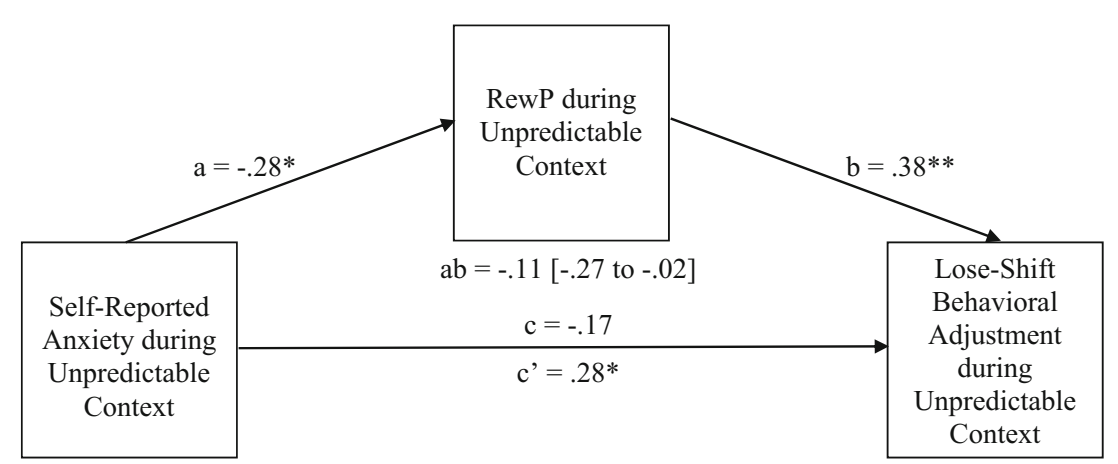

Fig. 3 Figure depicting the indirect relationship between self-reported anxiety, mediated through the RewP, on lose-shift behavioral adjustment during the unpredictable context. Coefficients are standardized regression weights. $\mathrm{a}=$ association between $\mathrm{X}$ and $\mathrm{M} ; \mathrm{b}=$ association between $\mathrm{M}$ and $\mathrm{Y} ; \mathrm{ab}=$ indirect effect; $\mathrm{c}=$ total effect of $\mathrm{X}$ on $\mathrm{Y} ; \mathrm{c}^{\prime}=$ direct effect of $\mathrm{X}$ on $\mathrm{Y} ; \mathrm{M}=$ mediator; RewP = reward positivity; $\mathrm{X}=$ independent variable; $\mathrm{Y}=$ dependent variable. ${ }^{*} p<.05$. $* * p<.01$ 
2015b), which are unpredictable, potentially threatening events (Hajcak, 2012). These results suggest that inhibitory IU has the same impact on both the ERN and RewP and may associated with decreased psychophysiological reactivity to motivationally salient stimuli in general (e.g., threat and reward). Prospective IU has been particularly associated with GAD (McEvoy \& Mahoney, 2012), a condition characterized by increased positive and negative emotional reactivity and poor emotion regulation (Mennin, Turk, Heimberg, \& Carmin, 2004). Indeed, in a separate investigation, greater prospective IU was associated with an increased startle reflex in anticipation of unpredictable threat (Nelson et al., 2015). These results suggest that, in contrast to inhibitory IU, high prospective IU is associated with increased psychophysiological reactivity to appetitive and aversive stimuli. Alternatively, high prospective IU individuals may have had more uncertain expectations about the outcome of each trial, and this in turn may have enhanced the RewP. This explanation is consistent with research indicating that the RewP is larger when outcomes are more unexpected and unpredictable (Hajcak, Holroyd, Moser, \& Simons, 2005; Holroyd \& Krigolson, 2007; Miltner et al., 1997; Nieuwenhuis, Holroyd, Mol, \& Coles, 2004). High prospective IU individuals may have also
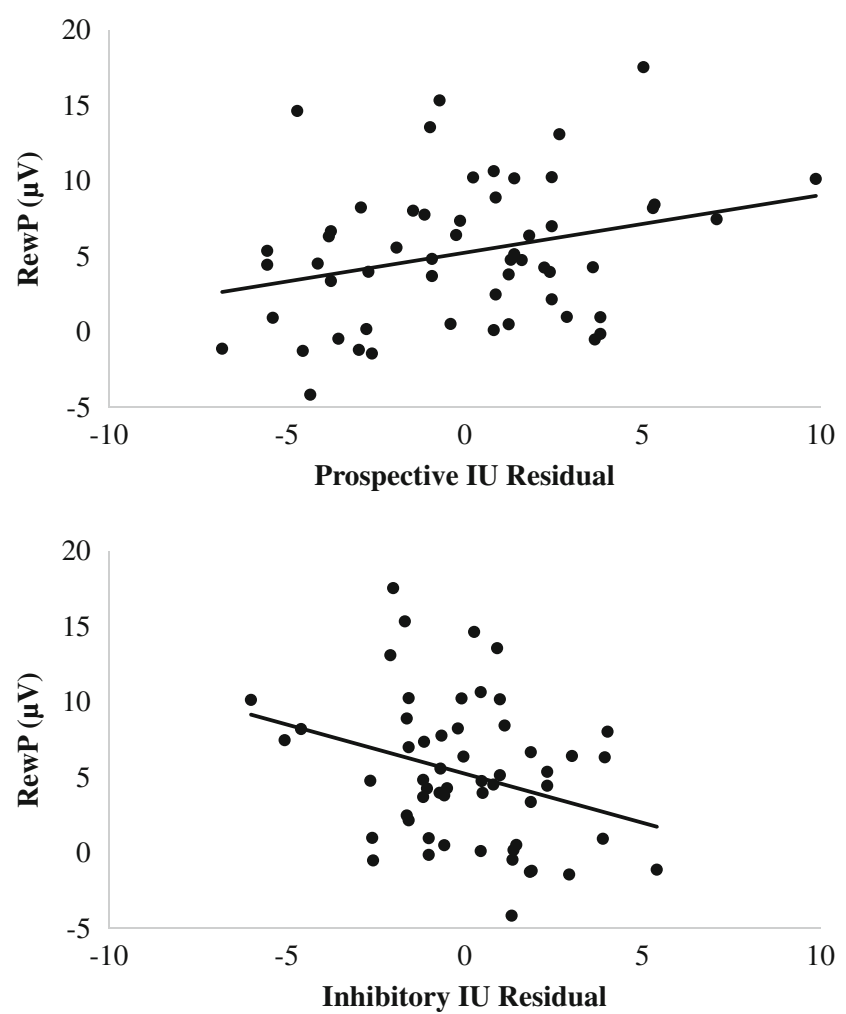

Fig. 4 Scatterplots depicting the association between prospective IU residual (controlling for inhibitory IU; top) and inhibitory IU residual (controlling for prospective IU; bottom) and the RewP (i.e., gain-loss) across both predictable and unpredictable contexts. IU = intolerance of uncertainty; RewP = reward-related positivity been more engaged in the task, which has been shown to increase the RewP (Yeung et al., 2005).

The IU subscales were associated with the RewP in the opposite direction, and this association only emerged when controlling for the other subscale. These results indicate that the IU subscales demonstrate reciprocal suppression effects (Watson, Clark, Chmielewski, \& Kotov, 2013), in which the predictors (i.e., inhibitory and prospective IU) are positively related to one another but oppositely correlated with the criterion (i.e., the RewP). There are a growing number of studies that have indicated opposing relationships between particular anxiety-related dimensions and reward responding (e.g., Kessel, Kujawa, Proudfit, \& Klein, in press). These findings are not particularly surprising given the substantial heterogeneity in anxiety disorders and depression (Mineka, Watson, \& Clark, 1998; Watson, 2005). Future studies should examine the IUS subscales as simultaneous predictors due to the potential for suppression effects.

The present study highlights the potential utility of examining transdiagnostic phenotypes in relation to neurobiological measures of reward and loss. Previous studies have found an attenuated RewP during a gambling task in individuals with high relative to low trait-like anxiety (Gu, Huang, \& Luo, 2010; Lange, Leue, \& Beauducel, 2012) and obsessive-compulsive symptoms (Simons, 2010). However, one study found that negative emotionality was associated with an enhanced RewP (Santesso et al., 2012) and other studies have found no association between anxiety and the RewP (Bress, Meyer, \& Hajcak, 2013). In this study, internalizing symptoms (anxiety, depression, stress, and worry) were not associated with the RewP across either the predictable or unpredictable context. These discrepant findings may be the result of focusing on broad anxiety symptoms and syndromes rather than more specific measures and mechanisms that span multiple disorders (e.g., IU). In short, IU is a potential transdiagnostic phenotype that may explain how individual differences in sensitivity to unpredictability may contribute to reward system dysfunction, which in turn contribute to the development and maintenance of psychopathology.

The RewP is posited to reflect activation of a reinforcement learning system that is used to adjust subsequent behavior (Holroyd \& Coles, 2002; Schultz, 2002). In the present study, there was a positive association between magnitude of the RewP and lose-shift behavioral adjustment during the unpredictable, but not predicable, context. In addition, self-reported anxiety elicited by the unpredictable context demonstrated both a positive direct relationship, but only after controlling for the RewP, and a negative indirect relationship with loseshift behavioral adjustment. The positive direct relationship may reflect increased hypervigilance, and subsequently more optimal behavioral performance, that often accompanies anxiety. In contrast, the negative indirect relationship suggests that the neural processing of rewards may be a mechanism 
linking anxiety and aberrations in approach-oriented behavior. There were no associations between the RewP and win-stay behavior or IU and either measure of behavioral adjustment. However, it is important to highlight that this study used a gambling paradigm (i.e., the doors task) that was designed to elicit the RewP but not optimized to measure reward-related behavioral modification. For example, there were an equal number of gain and loss trials, and participants may have realized their door selection was not related to trial outcome. Furthermore, the literature on the RewP and behavioral adjustment is mixed, with some studies reporting associations (Cohen \& Ranganath, 2007) and other studies finding no relationship (Chase, Swainson, Durham, Benham, \& Cools, 2011). Future attempts to replicate these findings should consider using experimental tasks that better assess reward-related behavioral adjustments (e.g., Cavanagh, 2015).

The present study had several limitations that warrant consideration. First, the study was limited to college students and the results may not generalize to other populations. Second, the sample had limited variability on the DASS-21, and this may have influenced the null association between anxiety, depression, stress, and the RewP. Third, the sample was primarily female, and it was underpowered to examined potential sex differences. Fourth, gain trials were reinforced at twice the rate $(\$ 0.50)$ of loss trials $(\$ 0.25)$ to equate their subjective value (Tversky \& Kahneman, 1981; Tversky \& Kahneman, 1992). This approach confounds valence (gain vs. loss) and magnitude of outcome (larger vs. smaller), and thus it is difficult to definitely determine whether any differences or associations with the RewP were due to either factor. Finally, the study did not include a no-tones condition, and it is unclear whether IU relates to the RewP in the absence of a contextual manipulation. Thus, future attempts to replicate these findings should also include a no-tones condition.

In conclusion, the present study found that a task-irrelevant unpredictable context can decrease the difference between ERP measures of reward and loss. In addition, individual differences in IU were associated with the neural processing of monetary outcomes, such that inhibitory IU was associated with a reduced RewP and prospective IU was associated with an enhanced RewP. These results add to growing evidence indicating that unpredictability is linked to core neural systems involved in reward processing (Dillon et al., 2009; Nelson, Shankman, \& Proudfit, 2014; Pizzagalli et al., 2007; Pryce et al., 2005).

\section{References}

Birrell, J., Meares, K., Wilkinson, A., \& Freeston, M. (2011). Toward a definition of intolerance of uncertainty: A review of factor analytical studies of the Intolerance of Uncertainty Scale. Clinical Psychology Review, 31, 1198-1208. doi:10.1016/j.cpr.2011.07.009

Bogdan, R., \& Pizzagalli, D. A. (2006). Acute stress reduces reward responsiveness: Implications for depression. Biological Psychiatry, 60, 1147-1154. doi:10.1016/j.biopsych.2006.03.037

Boswell, J. F., Thompson-Hollands, J., Farchione, T. J., \& Barlow, D. H. (2013). Intolerance of uncertainty: A common factor in the treatment of emotional disorders. Journal of Clinical Psychology, 69, 630 645. doi:10.1002/jclp. 21965

Bress, J. N., \& Hajcak, G. (2013). Self-report and behavioral measures of reward sensitivity predict the feedback negativity. Psychophysiology, 50, 610-616. doi:10.1111/psyp.12053

Bress, J. N., Meyer, A., \& Hajcak, G. (2013). Differentiating anxiety and depression in children and adolescents: Evidence from event-related brain potentials. Journal of Clinical Child and Adolescent Psychology, 37-41. doi:10.1080/15374416.2013.814544

Carleton, R. N., Norton, M. A. P. J., \& Asmundson, G. J. G. (2007). Fearing the unknown: A short version of the Intolerance of Uncertainty Scale. Journal of Anxiety Disorders, 21, 105-117. doi: 10.1016/j.janxdis.2006.03.014

Carleton, N. R., Mulvogue, M. K., Thibodeau, M. A., McCabe, R. E., Antony, M. M., \& Asmundson, G. J. G. (2012). Increasingly certain about uncertainty: Intolerance of uncertainty across anxiety and depression. Journal of Anxiety Disorders, 26, 468-479. doi:10.1016/j. janxdis.2012.01.011

Carlson, J. M., Foti, D., Mujica-Parodi, L. R., Harmon-Jones, E., \& Hajcak, G. (2011). Ventral striatal and medial prefrontal BOLD activation is correlated with reward-related electrocortical activity: A combined ERP and fMRI study. NeuroImage, 57, 1608-1616. doi:10.1016/j.neuroimage.2011.05.037

Cavanagh, J. F. (2015). Cortical delta activity reflects reward prediction error and related behavioral adjustments, but at different times. NeuroImage, 110, 205-216. doi:10.1016/j.neuroimage.2015.02.007

Chase, H. W., Swainson, R., Durham, L., Benham, L., \& Cools, R. (2011). Feedback-related negativity codes prediction error but not behavioral adjustment during probabilistic reversal learning. Journal of Cognitive Neuroscience, 23, 936-946. doi:10.1162/ jocn.2010.21456

Clark, L. A., \& Watson, D. (1991). Tripartite model of anxiety and depression: Psychometric evidence and taxonomic implications. Journal of Abnormal Psychology, 100, 316-336. doi:10.1037/ 0021-843X.100.3.316

Cohen, M. X., \& Ranganath, C. (2007). Reinforcement learning signals predict future decisions. The Journal of Neuroscience, 27, 371-378. doi:10.1523/JNEUROSCI.4421-06.2007

Dehaene, S., Posner, M. I., \& Tucker, D. M. (1994). Localization of a neural system for error detection and compensation. Psychological Science, 5, 303-305. doi:10.1111/j.1467-9280.1994.tb00630.x

Dillon, D. G., Holmes, A. J., Birk, J. L., Brooks, N., Lyons-Ruth, K., \& Pizzagalli, D. A. (2009). Childhood adversity is associated with left basal ganglia dysfunction during reward anticipation in adulthood. Biological Psychiatry, 66, 206-213. doi:10.1016/j.biopsych.2009. 02.019

Dugas, M. J., Gagnon, F., Ladouceur, R., \& Freeston, M. H. (1998). Generalized anxiety disorder: A preliminary test of a conceptual model. Behaviour Research and Therapy, 36, 215-226. doi:10. 1016/S0005-7967(97)00070-3

Dugas, M. J., Buhr, K., \& Ladouceur, R. (2004). The role of intolerance of uncertainty in etiology and maintenance. In R. G. Heimberg, C. L. Turk, \& D. S. Mennin (Eds.), Generalized anxiety disorder: Advances in research and practice (pp. 143-163). New York: Guilford Press.

Foti, D., Carlson, J. M., Sauder, C. L., \& Proudfit, G. H. (2014). Reward dysfunction in major depression: Multimodal neuroimaging 
evidence for refining the melancholic phenotype. NeuroImage, 101, 50-58. doi:10.1016/j.neuroimage.2014.06.058

Freeston, M. H., Rhéaume, J., Letarte, H., Dugas, M. J., \& Ladouceur, R. (1994). Why do people worry? Personality and Individual Differences, 17, 791-802. doi:10.1016/0191-8869(94)90048-5

Frewen, P. A., Dozois, D. J., Joanisse, M. F., \& Neufeld, R. W. (2008). Selective attention to threat versus reward: Meta-analysis and neural-network modeling of the dot-probe task. Clinical Psychology Review, 28, 307-337. doi:10.1016/j.cpr.2007.05.006

Gratton, G., Coles, M. G. H., \& Donchin, E. (1983). A new method for off-line removal of ocular artifact. Electroencephalography and Clinical Neurophysiology, 55, 468-484. doi:10.1016/00134694(83)90135-9

Grillon, C., Baas, J. M. P., Cornwell, B., \& Johnson, L. (2006). Context conditioning and behavioral avoidance in a virtual reality environment: Effect of predictability. Biological Psychiatry, 60, 752-759. doi:10.1016/j.biopsych.2006.03.072

Grupe, D. W., \& Nitschke, J. B. (2013). Uncertainty and anticipation in anxiety: An integrated neurobiological and psychological perspective. Nature Reviews Neuroscience, 14, 488-501. doi:10.1038/ nrn3524

Gu, R., Huang, Y. X., \& Luo, Y. J. (2010). Anxiety and feedback negativity. Psychophysiology, 47, 961-967. doi:10.1111/j.1469-8986. 2010.00997.x

Hajcak, G. (2012). What we've learned from mistakes: Insights from error-related brain activity. Current Directions in Psychological Science, 21, 101-106. doi:10.1177/0963721412436809

Hajcak, G., Holroyd, C. B., Moser, J. S., \& Simons, R. F. (2005). Brain potentials associated with expected and unexpected good and bad outcomes. Psychophysiology, 42, 161-170. doi:10.1111/j.14698986.2005.00278.x

Hajcak, G., Moser, J. S., Holroyd, C. B., \& Simons, R. F. (2006). The feedback-related negativity reflects the binary evaluation of good versus bad outcomes. Biological Psychology, 71, 148-154. doi:10. 1016/j.biopsycho.2005.04.001

Hajcak, G., Moser, J. S., Holroyd, C. B., \& Simons, R. F. (2007). It's worse than you thought: The feedback negativity and violations of reward prediction in gambling tasks. Psychophysiology, 44, 905 912. doi:10.1111/j.1469-8986.2007.00567.x

Harmon-Jones, E., \& Harmon-Jones, C. (2002). Testing the action-based model of cognitive dissonance: The effect of action orientation on postdecisional attitudes. Personality and Social Psychology Bulletin, 28, 711-723. doi:10.1177/0146167202289001

Hayes, A. (2013). Introduction to mediation, moderation, and conditional process analysis. New York: Guilford Press.

Heine, S. J., Proulx, T., \& Vohs, K. D. (2006). The meaning maintenance model: On the coherence of social motivations. Personality and Social Psychology Review, 10, 88-110. doi:10.1207/ s15327957pspr1002_1

Herry, C., Bach, D. R., Esposito, F., Di Salle, F., Perrig, W. J., Scheffler, K., . . . Seifritz, E. (2007). Processing of temporal unpredictability in human and animal amygdala. The Journal of Neuroscience, 27, 5958-5966. doi:10.1523/JNEUROSCI.5218-06.2007

Holroyd, C. B., \& Coles, M. G. H. (2002). The neural basis of human error processing: Reinforcement learning, dopamine, and the errorrelated negativity. Psychological Review, 109, 679-709. doi:10. 1037/0033-295X.109.4.679

Holroyd, C. B., \& Krigolson, O. E. (2007). Reward prediction error signals associated with a modified time estimation task. Psychophysiology, 44, 913-917. doi:10.1111/j.1469-8986.2007. 00561.x

Holroyd, C. B., Dien, J., \& Coles, M. G. H. (1998). Error-related scalp potentials elicited by hand and foot movements: Evidence for an output-independent error-processing system in humans.
Neuroscience Letters, 242, 65-68. doi:10.1016/S0304-3940(98) 00035-4

Holroyd, C. B., Hajcak, G., \& Larsen, J. T. (2006). The good, the bad and the neutral: Electrophysiological responses to feedback stimuli. Brain Research, 1105, 93-101. doi:10.1016/j.brainres.2005.12.015

Holroyd, C. B., Pakzad-Vaezi, K. L., \& Krigolson, O. E. (2008). The feedback correct-related positivity: Sensitivity of the event-related brain potential to unexpected positive feedback. Psychophysiology, 45, 688-697. doi:10.1111/j.1469-8986.2008.00668.x

Jackson, F., Nelson, B. D., \& Proudfit, G. H. (2015a). In an uncertain world, errors are more aversive: Evidence from the error-related negativity. Emotion, 15, 12-16. doi:10.1037/emo0000020

Jackson, F., Nelson, B. D., \& Hajcak, G. (2015b). Intolerance of uncertainty is associated with error-related brain activity. Manuscript under review.

Kessel, E. M., Kujawa, A., Proudfit, G. H., \& Klein, D. N. (in press). Neural reactivity to monetary rewards and losses differentiates social from generalized anxiety in children. Journal of Child Psychology and Psychiatry. doi:10.1111/jcpp.12355

Lange, S., Leue, A., \& Beauducel, A. (2012). Behavioral approach and reward processing: Results on feedback-related negativity and $\mathrm{P} 3$ component. Biological Psychology, 89, 416-425. doi:10.1016/j. biopsycho.2011.12.004

Lejuez, C. W., Eifert, G. H., Zvolensky, M. J., \& Richards, J. B. (2000). Preference between onset predictable and unpredictable administrations of $20 \%$ carbon-dioxide-enriched air: Implications for better understanding the etiology and treatment of panic disorder. Journal of Experimental Psychology: Applied, 6, 349-358. doi:10. 1037/1076-898X.6.4.349

Lemos, J. C., Wanat, M. J., Smith, J. S., Reyes, B. A. S., Hollon, N. G., Van Bockstaele, E. J., . . . Phillips, P. E. M. (2012). Severe stress switches CRF action in the nucleus accumbens from appetitive to aversive. Nature, 490, 402-406. doi:10.1038/nature11436

Lerner, M. J. (1980). Belief in a just world: A fundamental delusion. New York: Plenum Press. doi:10.1007/978-1-4899-0448-5

Lovibond, S. H., \& Lovibond, P. F. (1995). Manual for the Depression Anxiety Stress Scales. New South Wales: Psychology Foundation of Australia. doi:10.1016/0005-7967(94)00075-U

MacKinnon, D. P., Lockwood, C. M., Hoffman, J. M., West, S. G., \& Sheets, V. (2002). A comparison of methods to test mediation and other intervening variable effects. Psychological Methods, 7, 83104. doi:10.1037/1082-989X.7.1.83

MacKinnon, D. P., Lockwood, C. M., \& Williams, J. (2004). Confidence limits for the indirect effect: Distribution of the product and resampling methods. Multivariate Behavioral Research, 39, 99-128. doi: $10.1207 / \mathrm{s} 15327906 \mathrm{mbr} 39014$

Mahoney, A. E. J., \& McEvoy, P. M. (2012). A transdiagnostic examination of intolerance of uncertainty across anxiety and depressive disorders. Cognitive Behaviour Therapy, 41, 212-222. doi:10.1080/ 16506073.2011 .622130

McEvoy, P. M., \& Mahoney, A. E. J. (2012). To be sure, to be sure: Intolerance of uncertainty mediates symptoms of various anxiety disorders and depression. Behavior Therapy, 43, 533-545. doi:10. 1016/j.beth.2011.02.007

McGregor, I., Prentice, M. S., \& Nash, K. A. (2009). Personal uncertainty management by reactive approach motivation. Psychological Inquiry, 20, 225-229. doi:10.1080/10478400903333460

Mennin, D. S., Turk, C. L., Heimberg, R. G., \& Carmin, C. (2004). Focusing on the regulation of emotion: A new direction for conceptualizing generalized anxiety disorder. In M. A. Reinecke \& D. A. Clark (Eds.), Cognitive therapy over the lifespan: evidence and practice (pp. 60-89). New York: Cambridge University Press.

Meyer, T. J., Miller, M. L., Metzger, R. L., \& Borkovec, T. D. (1990). Development and validation of the Penn State Worry Questionnaire. 
Behaviour Research and Therapy, 28, 487-495. doi:10.1016/00057967(90)90135-6

Miltner, W. H. R., Braun, C. H., \& Coles, M. G. H. (1997). Event-related brain potentials following incorrect feedback in a time-estimation task: Evidence for a "generic" neural system for error detection. Journal of Cognitive Neuroscience, 9, 788-798. doi:10.1162/jocn. 1997.9.6.788

Mineka, S., Watson, D., \& Clark, L. A. (1998). Comorbidity of anxiety and unipolar mood disorders. Annual Review of Psychology, 49, 377-412. doi:10.1146/annurev.psych.49.1.377

Nelson, B. D., \& Shankman, S. A. (2011). Does intolerance of uncertainty predict anticipatory startle responses to uncertain threat? International Journal of Psychophysiology, 81, 107-115. doi:10. 1016/j.ijpsycho.2011.05.003

Nelson, B. D., Shankman, S. A., \& Proudfit, G. H. (2014). Intolerance of uncertainty mediates reduced reward anticipation in major depressive disorder. Journal of Affective Disorders, 158, 108-113. doi:10. 1016/j.jad.2014.02.014

Nelson, B. D., Liu, H., Sarapas, C., \& Shankman, S. A. (2015). Intolerance of uncertainty mediates the relationship between panic and the startle reflex in anticipation of unpredictable threat. Manuscript under review.

Nieuwenhuis, S., Holroyd, C. B., Mol, N., \& Coles, M. G. H. (2004). Reinforcement-related brain potentials from medial frontal cortex: Origins and functional significance. Neuroscience and Biobehavioral Reviews, 28, 441-448. doi:10.1016/j.neubiorev. 2004.05.003

Papp, M., Willner, P., \& Muscat, R. (1991). An animal model of anhedonia: Attenuation of sucrose consumption and place preference conditioning by chronic unpredictable mild stress. Psychopharmacology, 104, 255-259. doi:10.1007/BF02244188

Pizzagalli, D. A., Bogdan, R., Ratner, K. G., \& Jahn, A. L. (2007). Increased perceived stress is associated with blunted hedonic capacity: Potential implications for depression research. Behaviour Research and Therapy, 45, 2742-2753. doi:10.1016/j.brat.2007. 07.013

Poli, S., Sarlo, M., Bortoletto, M., Buodo, G., \& Palomba, D. (2007). Stimulus-preceding negativity and heart rate changes in anticipation of affective pictures. International Journal of Psychophysiology, 65, 32-39. doi:10.1016/j.ijpsycho.2007.02.008

Porcelli, A. J., Lewis, A. H., \& Delgado, M. R. (2012). Acute stress influences neural circuits of reward processing. Frontiers in Neuroscience, 6, 157. doi:10.3389/fnins.2012.00157

Preacher, K. J., \& Hayes, A. F. (2004). SPSS and SAS procedures for estimating indirect effects in simple mediation models. Behavior Research Methods, Instruments, \& Computers, 36, 717-731. doi: 10.3758/BF03206553

Proudfit, G. H. (2015). The reward positivity: From basic research on reward to a biomarker for depression. Psychophysiology, 52, 449459. doi:10.1111/psyp. 12370
Pryce, C., Rüedi-Bettschen, D., \& Dettling, A. (2005). Long-term effects of early-life environmental manipulations in rodents and primates: Potential animal models in depression research. Neuroscience and Biobehavioral Reviews, 29, 649-674. doi:10.1016/j.neubiorev. 2005.03.011

Robichaud, M. (2013). Cognitive behavior therapy targeting intolerance of uncertainty: Application to a clinical case of generalized anxiety disorder. Cognitive and Behavioral Practice, 20, 251-263. doi:10. 1016/j.cbpra.2012.09.001

Santesso, D. L., Bogdan, R., Birk, J. L., Goetz, E. L., Holmes, A. J., \& Pizzagalli, D. A. (2012). Neural responses to negative feedback are related to negative emotionality in healthy adults. Social Cognitive and Affective Neuroscience, 7, 794-803. doi:10.1093/scan/nsr054

Schultz, W. (2002). Getting formal with dopamine and reward. Neuron, 36, 241-263. doi:10.1016/S0896-6273(02)00967-4

Simons, R. F. (2010). The way of our errors: Theme and variations. Psychophysiology, 47, 1-14. doi:10.1111/j.1469-8986.2009.00929.x

Tolin, D. F., Abramowitz, J. S., Brigidi, B. D., \& Foa, E. B. (2003). Intolerance of uncertainty in obsessive-compulsive disorder. Journal of Anxiety Disorders, 17, 233-242. doi:10.1016/S08876185(02)00182-2

Treadway, M. T., Buckholtz, J. W., \& Zald, D. H. (2013). Perceived stress predicts altered reward and loss feedback processing in medial prefrontal cortex. Frontiers in Human Neuroscience, 7, 180. doi:10. 3389/fnhum.2013.00180

Tversky, A., \& Kahneman, D. (1981). The framing of decisions and the psychology of choice. Science, 211, 453-458. doi:10.1126/science. 7455683

Tversky, A., \& Kahneman, D. (1992). Advances in prospect theory: Cumulative representation of uncertainty. Journal of Risk and Uncertainty, 5, 297-323. doi:10.1007/BF00122574

Van der Heiden, C., Muris, P., \& van der Molen, H. T. (2012). Randomized controlled trial on the effectiveness of metacognitive therapy and intolerance-of-uncertainty therapy for generalized anxiety disorder. Behaviour Research and Therapy, 50, 100-109. doi: 10.1016/j.brat.2011.12.005

Watson, D. (2005). Rethinking the mood and anxiety disorders: A quantitative hierarchical model for DSM-V. Journal of Abnormal Psychology, 114, 522-536. doi:10.1037/0021-843X.114.4.522

Watson, D., Clark, L. A., Chmielewski, M., \& Kotov, R. (2013). The value of suppressor effects in explicating the construct validity of symptom measures. Psychological Assessment, 25, 929-941. doi: 10.1037/a0032781

Willner, P., Towell, A., Sampson, D., Sophokleous, S., \& Muscat, R. (1987). Reduction of sucrose preference by chronic unpredictable mild stress, and its restoration by a tricyclic antidepressant. Psychopharmacology, 93, 358-364. doi:10.1007/BF00187257

Yeung, N., Holroyd, C. B., \& Cohen, J. D. (2005). ERP correlates of feedback and reward processing in the presence and absence of response choice. Cerebral Cortex, 15, 535-544. doi:10.1093/ cercor/bhh153 